\title{
Hymenolepis nana
}

National Cancer Institute

\section{Source}

National Cancer Institute. Hymenolepis nana. NCI Thesaurus. Code C122323.

A species of tapeworm in the family Hymenolepididae. The lifecycle of $\mathrm{H}$. nana involves insects as the intermediate host and rodents or humans as the definitive host. Eggs may also be transferred directly from person to person. 